\title{
An open-label randomised controlled trial on the efficacy of adding intranasal fentanyl to intravenous tramadol in patients with moderate to severe pain following acute musculoskeletal injuries
}

Keng Sheng $\underline{C h e w}^{1}$, MD, MMed, Abdul Hafiz Shaharudin ${ }^{1}$, MD

\begin{abstract}
INTRODUCTION The use of intranasal fentanyl as an alternative type of analgesia has been shown to be effective in paediatric populations and prehospital settings. There are a limited number of studies on the use of intranasal fentanyl in adult patients in emergency settings.

METHODS An open-label study was conducted to evaluate the effectiveness of the addition of $1.5 \mathrm{mcg} / \mathrm{kg}$ intranasal fentanyl to $2 \mathrm{mg} / \mathrm{kg}$ intravenous tramadol (fentanyl + tramadol arm, $\mathrm{n}=10$ ) as compared to the administration of $2 \mathrm{mg} / \mathrm{kg}$ intravenous tramadol alone (tramadol-only arm, $n=10$ ) in adult patients with moderate to severe pain due to acute musculoskeletal injuries.

RESULTS When analysed using the independent $t$-test, the difference between the mean visual analogue scale scores pre-intervention and ten minutes post-intervention was $29.8 \pm 8.4 \mathrm{~mm}$ in the fentanyl + tramadol arm and $19.6 \pm 9.7 \mathrm{~mm}$ in the tramadol-only arm $(\mathrm{t}[18]=2.515, \mathrm{p}=0.022,95 \%$ confidence interval $1.68-18.72 \mathrm{~mm})$. A statistically significant, albeit transient, reduction in the ten-minute post-intervention mean arterial pressure was noted in the fentanyl + tramadol arm as compared to the tramadol-only arm $(13.35 \mathrm{mmHg}$ vs. $7.65 \mathrm{mmHg}$; using Mann-Whitney $U$ test with $U$-value 21.5 , $p=0.029, r=0.48)$. There was a higher incidence of transient dizziness ten minutes after intervention among the patients in the fentanyl + tramadol arm.

CONCLUSION Although effective, intranasal fentanyl may not be appropriate for routine use in adult patients, as it could result in a significant reduction in blood pressure.
\end{abstract}

Keywords: fentanyl, intranasal drug administration, tramadol

\section{INTRODUCTION}

Although pain is a common presentation in emergency departments (EDs), (1) lack of pain control or 'oligoanalgesia' frequently occurs. ${ }^{(2)}$ In a crowded ED, time to analgesia is often prolonged $^{(3)}$ and this can be detrimental, as the quality of pain management affects patient outcomes.

One of the factors that improves the timing of pain management is the route of analgesia administration. Occasionally, clinicians may have difficulty in establishing an intravenous line, resulting in a delay in drug administration. Administering analgesia via the intranasal route has recently been advocated as an alternative method to overcome the problem of delayed drug administration. ${ }^{(4)}$ In properly selected patients, use of the intranasal route reduces the time from drug administration to the onset of drug action. It can also help to alleviate manpower constraints and eliminate needlestick exposure risk and injection pain, compared to drug administration via injections. ${ }^{(5)}$ The intranasal route enables rapid absorption of the administered drug because the nasal mucosa is highly vascularised; only two cell layers separate the nasal lumen from the nasal mucosa's blood vascular system. ${ }^{(6)}$ Furthermore, this route affords a large surface area (150-180 $\left.\mathrm{m}^{2}\right)^{(7)}$ for drug delivery and eliminates first-pass metabolism, ${ }^{(8)}$ allowing the drug to enter the cerebrospinal fluid via the olfactory mucosa for immediate therapeutic effect. ${ }^{(9)}$ For example, intranasal fentanyl has been shown to achieve therapeutic serum levels within two minutes of administration. ${ }^{(10)}$

Tramadol, a synthetic opioid of the aminocyclohexanol group, has been shown to possess an analgesic potency equivalent to that of pethidine. ${ }^{(11)}$ Fentanyl is a synthetic phenylpiperidine derivative whose analgesic potency is 50-80 times that of morphine. ${ }^{(12)}$ Fentanyl also has a rapid onset of action (within 6-8 minutes following intranasal administration) due to its high lipid solubility. ${ }^{(13)}$ Studies conducted in prehospital settings have shown that intranasal fentanyl is as effective an analgesic as intravenous morphine in adult ${ }^{(14)}$ and paediatric patients. ${ }^{(15)}$

Although the evidence regarding the use of intranasal fentanyl in EDs is limited, the few published studies show promising results. In one study, intranasal fentanyl was shown to be as effective an analgesic as intramuscular morphine in children presenting to the ED. ${ }^{(16)}$ In another study conducted in an ED setting, intranasal fentanyl was shown to be comparable to intravenous morphine in reducing pain in a paediatric population that had acute long bone fractures. ${ }^{(17)}$ Yet another study showed that it provides effective analgesia for paediatric patients with painful orthopaedic traumas. ${ }^{(18)}$ While the use of intranasal fentanyl in the ED has been shown to be effective in paediatric populations, ${ }^{(9)}$ there are

${ }^{1}$ Emergency Medicine Department, School of Medical Sciences, Universiti Sains Malaysia, Malaysia

Correspondence: Dr Keng Sheng Chew, Senior Lecturer and Emergency Physician, Emergency Medicine Department, School of Medical Sciences, Universiti Sains Malaysia, Health Campus, 16150 Kubang Kerian, Kelantan, Malaysia. cksheng74@usm.my 
a limited number of studies regarding its use in adult patients in emergency settings. Thus, the present study aimed to examine the efficacy of the use of intranasal fentanyl in adult patients presenting to the ED of a tertiary hospital in Malaysia.

\section{METHODS}

This was a prospective, randomised, open-label study conducted to evaluate the effectiveness of using intranasal fentanyl in addition to intravenous tramadol for patients with moderate to severe pain due to acute musculoskeletal injuries. This study was approved by the Human Research Ethics Committee of Universiti Sains Malaysia, Malaysia.

This study was conducted in the ED of Hospital Universiti Sains Malaysia, a 750-bed tertiary referral centre and teaching hospital located in the east coast of Peninsular Malaysia. Data was obtained from a convenience sample that consisted of all adult patients (i.e. aged $\geq 18$ years) who presented between January and April 2014 within the stipulated study period during office hours with moderate to severe pain due to musculoskeletal injuries. The primary outcome of this study was subjective improvement of pain severity, measured using a visual analogue scale (VAS) at ten minutes after intervention. The secondary outcomes were changes in mean arterial pressure and heart rate before and ten minutes after intervention, as well as the incidence of other side effects.

Patients with polytrauma or significant comorbidities (e.g. hypertension, diabetes mellitus and cardiovascular diseases), and patients who were pregnant or had haemodynamic instability requiring resuscitation and stabilisation were excluded. As the study involved the administration of drugs via the intranasal route, patients with rhinopharyngitis or any intranasal pathology were excluded. Patients without the mental capacity to evaluate pain severity or with visual impairments that made it difficult or impossible for them to use the VAS were also excluded. We also excluded patients who had received any form of opioid before the intervention or had a history of opioid allergy.

The required sample size was estimated based on the twomeans formula, ${ }^{(19)}$ using the independent $t$-test with $\alpha=0.05$ and power $=0.8$. Using a standard deviation of $15 \mathrm{~mm}$ with an effect size for VAS ratings of $20 \mathrm{~mm}$ and accounting for a $10 \%$ dropout rate, the sample size needed was estimated to be ten patients per treatment arm. The effect size for VAS ratings was taken to be $20 \mathrm{~mm}$ because this value is considered to be clinically important, based on a previous study that was conducted on the same patient population. ${ }^{(20)}$

Each patient's pain severity was assessed using VAS by the clinician who managed the patient. The patient was asked to place a mark (i.e. ' $X$ ') to represent the severity of the pain. Moderate pain was defined as a VAS rating of $45-74 \mathrm{~mm}$, while severe pain was defined as a VAS rating of $75-100 \mathrm{~mm} .{ }^{(21)}$ Written consent was obtained if the patient fulfilled the study criteria. After pain severity was measured and written consent obtained, the clinician would alert one of the investigators, who would then allocate the patient to one of the treatment arms (either intranasal fentanyl and intravenous tramadol, or intravenous tramadol only). Allocation was done by asking the patient to select a piece of paper (stating which treatment the patient would receive) from an opaque envelope. Patients allocated to one treatment arm were blinded to the type of treatment received by patients in the other treatment arm.

Intranasal delivery of fentanyl was performed using an intranasal mucosal atomisation device (LMA® MAD Nasal ${ }^{\mathrm{TM}}$; Teleflex, Wayne, PA, USA). The comparative administration of a placebo via the intranasal route for the patients in the tramadol-only arm was not approved by our institution's Human Research Ethics Committee, due to ethical considerations and the unconventionality of the use of the intranasal route to administer analgesics.

For the patients who received intranasal fentanyl in addition to intravenous tramadol, intranasal fentanyl was first administered at a dose of $1.5 \mathrm{mcg} / \mathrm{kg}$ (solution concentration $50 \mathrm{mcg} / \mathrm{mL}$ ) using the LMA MAD Nasal intranasal mucosal atomisation device. With the patient propped up at an inclination of $45^{\circ}$, fentanyl was delivered in increments of $0.25 \mathrm{~mL}$, via slow push, into the right and left nostrils (alternately) for five minutes until the calculated dose was achieved. For all patients in this treatment arm, the intranasal fentanyl was administered by one of the investigators of the study. Intravenous access was established within two minutes after the administration of intranasal fentanyl. Intravenous metoclopramide $10 \mathrm{mg}$ was administered as an antiemetic for all patients, followed by slow bolus delivery of intravenous tramadol $2 \mathrm{mg} / \mathrm{kg}$ over one minute.

For the patients who received intravenous tramadol alone, intravenous metoclopramide $10 \mathrm{mg}$ was similarly given, followed by intravenous tramadol $2 \mathrm{mg} / \mathrm{kg}$. The time when all treatment had been given (fentanyl and tramadol, or tramadol alone) was marked as 'Time 0 '. The patient would then be observed for ten minutes. At the end of the ten minutes (i.e. 'Time 10'), the intensity of the patient's pain was assessed again using the same VAS. Throughout the observation period, the patients in both treatment arms were monitored by the investigators and instructed to report any side effects experienced. After the ten minutes (i.e. 'Time 10') had passed, the patients would be offered more pain relief, if necessary. All patients were observed for at least another 1-2 hours in the ED, during which their half-hourly blood pressure was recorded. Thereafter, definitive management of the patients was determined by the independent clinicians who managed them.

\section{RESULTS}

A total of 20 patients, aged 18-65 years, who met the study's inclusion criteria were invited to participate, and all voluntarily agreed to participate. Of these patients, ten were allocated to the fentanyl + tramadol arm and ten were allocated to the tramadolonly arm. 4 (40\%) patients and 3 (30\%) patients sustained bone fractures in the fentanyl + tramadol arm and the tramadol-only arm, respectively. The remaining patients had soft tissue injuries without bone fractures. The characteristics of the patients in both treatment arms are shown in Table I. Changes in the intensity of pain experienced by the patients before and after the respective interventions are shown in Fig. 1. 


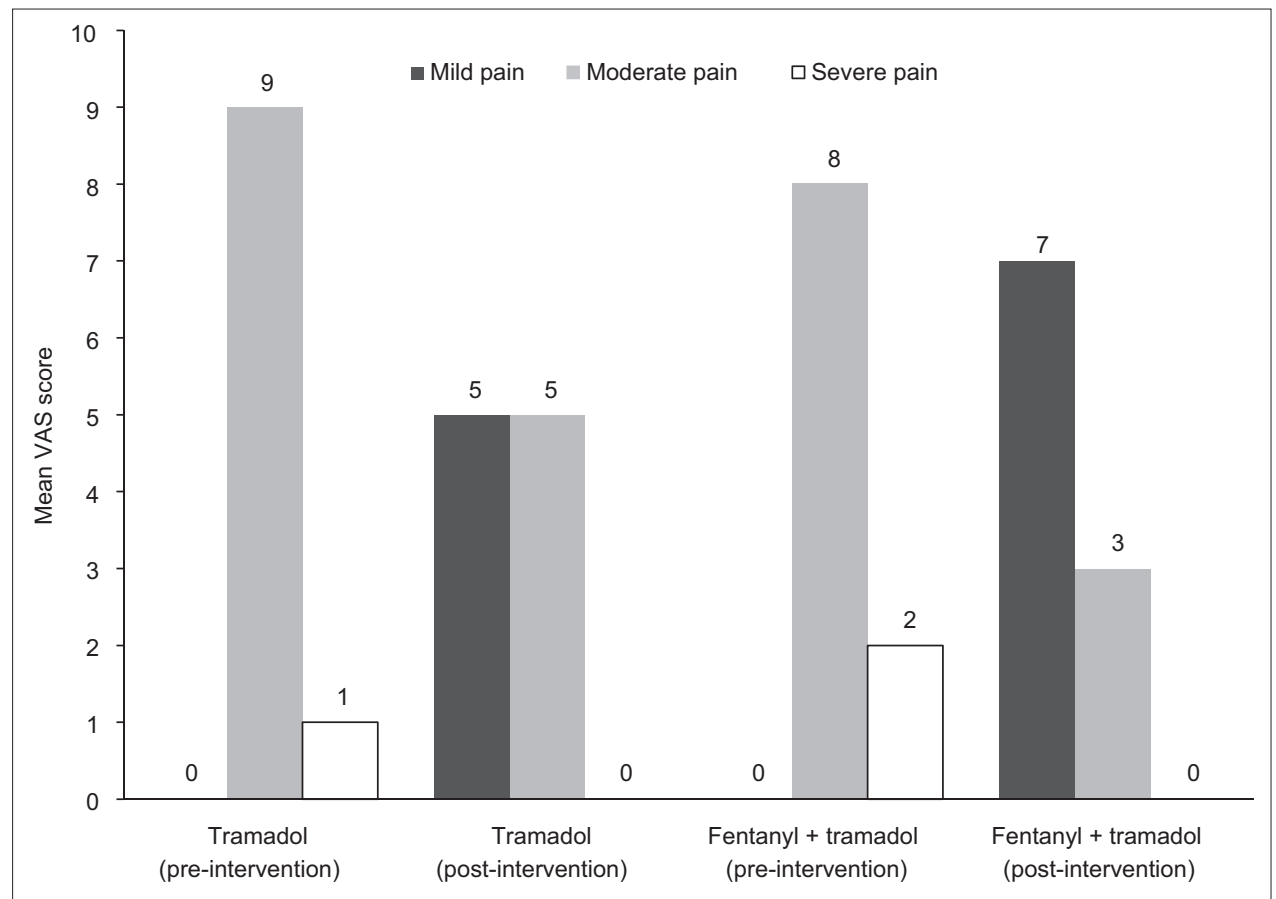

Fig. 1 Bar graph shows the intensity of pain experienced by the patients before and after intervention with tramadol alone or with fentanyl and tramadol. VAS: visual analogue scale

Table I. Characteristics of the patients included in the present study $(n=20)$

\begin{tabular}{|c|c|c|}
\hline \multirow[t]{2}{*}{ Characteristic } & \multicolumn{2}{|c|}{ No. (\%) } \\
\hline & Fentanyl + tramadol $(n=10)$ & Tramadol only $(n=10)$ \\
\hline \multicolumn{3}{|l|}{ Gender } \\
\hline Male & $8(80.0)$ & $8(80.0)$ \\
\hline Female & $2(20.0)$ & $2(20.0)$ \\
\hline \multicolumn{3}{|l|}{ Type of injury } \\
\hline Soft tissue injury with bone fractures & $4(40.0)$ & $3(30.0)$ \\
\hline Soft tissue injury without bone fractures & $6(60.0)$ & $7(70.0)$ \\
\hline \multicolumn{3}{|l|}{ Pre-intervention vital sign* } \\
\hline Systolic BP (mmHg) & $128.70 \pm 6.50$ & $121.60 \pm 9.73$ \\
\hline Diastolic BP (mmHg) & $73.40 \pm 6.59$ & $67.20 \pm 4.64$ \\
\hline Oxygen saturation (\%) & $99.30 \pm 1.34$ & $99.60 \pm 0.70$ \\
\hline Heart rate $(\mathrm{bpm})$ & $86.70 \pm 17.73$ & $85.50 \pm 16.90$ \\
\hline \multicolumn{3}{|l|}{ Post-intervention vital sign* } \\
\hline Systolic BP (mmHg) & $118.90 \pm 7.4$ & $118.10 \pm 8.2$ \\
\hline Diastolic BP (mmHg) & $68.00 \pm 5.89$ & $67.20 \pm 4.64$ \\
\hline Oxygen saturation (\%) & $99.50 \pm 0.97$ & $99.5 \pm 0.71$ \\
\hline Heart rate (bpm) & $78.80 \pm 14.64$ & $78.70 \pm 11.39$ \\
\hline \multicolumn{3}{|l|}{ Adverse effect due to intervention } \\
\hline Nausea & 0 & 0 \\
\hline Vomiting & 0 & 0 \\
\hline Dizziness & $3(30.0)$ & $4(40.0)$ \\
\hline Sleepiness & $8(80.0)$ & $5(50.0)$ \\
\hline
\end{tabular}

*Data presented as mean \pm standard deviation. BP: blood pressure

In both arms, the difference between the mean VAS score at 'Time 0 ' and 'Time $10^{\prime}$ was analysed using independent $t$-test. Shapiro-Wilk test ( $p>0.05$ ) and visual inspections of Q-Q plots, box plots and histograms showed that the difference in the mean VAS scores was approximately normally distributed for both treatment arms. In the fentanyl + tramadol arm, there was a skewness of -0.37 with a standard error (SE) of \pm 0.69 (z-score: -0.54 ) and a kurtosis of -1.18 with an SE of \pm 1.33 ( $z$-score: -0.88 ); in the tramadol-only arm, there was a skewness of 0.09 with an SE of \pm 0.69 (z-score: 0.13 ) and a kurtosis of -0.27 with an SE of \pm 1.33 (z-score: -0.20 ). As absolute z-scores for skewness and kurtosis were within 1.96, the sample was normally distributed. ${ }^{(22)}$ 
The improvement in the mean VAS scores of the patients in the fentanyl + tramadol arm and the tramadol-only arm was $29.8 \pm 8.4 \mathrm{~mm}$ and $19.6 \pm 9.7 \mathrm{~mm}$, respectively. This was a difference of $10.2 \pm 4.1 \mathrm{~mm}$ (SE of mean) at a 95\% confidence interval of 1.68-18.72 $\mathrm{mm}$ and was statistically significant $(\mathrm{t}[18]=2.515, \mathrm{p}=0.022)$. Levene's test was performed to verify the homogeneity of the variances in the sample $(p>0.05)$. To control for the confounding effects of the covariates of age, gender and baseline VAS score, a one-way analysis of covariance was performed. The analysis showed that after controlling for these covariates, there was still a statistically significant difference between the improvement in the mean VAS scores of the two treatment arms $(F[1,15]=4.592, p=0.049)$. The effect size (partial $\eta^{2}$ ) was 0.234 and homogeneity of regression was assumed.

Mean arterial pressure reduction, which is defined as the difference between the pre-intervention mean arterial pressure and the post-intervention mean arterial pressure, showed a nonparametric distribution. The reduction in mean arterial pressure was greater in the fentanyl + tramadol arm as compared to the tramadol-only arm (mean rank reduction in mean arterial pressure was $13.35 \mathrm{mmHg}$ in the fentanyl + tramadol arm and $7.65 \mathrm{mmHg}$ in the tramadol-only arm; $U$-value $=21.5, p=0.029, r=0.48$ ). The critical value for two-tailed $\alpha$ at 0.05 was 23 , which was larger than the $U$-value. Homogeneity of variance was assumed using the non-parametric Levene's test with $p>0.05$.

Categorical analysis using Fisher's exact test was performed to compare the incidence of the side effect of dizziness between the two treatment arms. Fisher's exact test was used as the total sample size was 20, with two cells (50\%) having an expected count $<5$. The side effect of dizziness occurred in $3(30.0 \%)$ patients in the fentanyl + tramadol arm and $4(40.0 \%)$ patients in the tramadol-only arm, but the difference was not statistically significant $(p=1.0)$. Fisher's exact test was also performed to compare the incidence of the side effect of sleepiness between the two treatment arms. Sleepiness occurred in $8(80.0 \%)$ patients in the fentanyl + tramadol arm and $5(50.0 \%)$ patients in the tramadol-only arm; the difference between the two treatment arms was similarly not statistically significant $(p=0.350)$.

All patients in the fentanyl + tramadol arm tolerated the administration of intranasal fentanyl, with none complaining of nasal irritation after administration. None of the patients in both treatment arms requested for more analgesia after the initial ten minutes of observation.

\section{DISCUSSION}

Not all medications are suitable for delivery via the intranasal route. Only drugs that have high potency and are available in concentrated preparations are suitable, as volume $>1 \mathrm{~mL}$ per nostril may not be delivered reliably due to mucosal surface saturation and runoff from the nasal cavity. ${ }^{(5)}$ As fentanyl has high lipophilicity and a potency that is 50-100 times that of morphine, it is suitable for intranasal administration. ${ }^{(9)}$

In the present study, we found that the addition of intranasal fentanyl to intravenous tramadol resulted in statistically significant pain reduction as compared to treatment with intravenous tramadol alone. This was evidenced by a greater reduction in the mean VAS score, at ten minutes after intervention, among the patients in the fentanyl + tramadol arm as compared to the patients in the tramadol-only arm. This finding indicates that intranasal fentanyl could be used as an effective analgesic adjunct, especially since it is well tolerated, relatively noninvasive and only requires a mucosal atomiser device for delivery. However, intranasal fentanyl may not be appropriate for routine use in adult populations just yet, as this study showed that its use as an adjunct resulted in a statistically significant, albeit transient, reduction of blood pressure as well as dizziness during the ten minutes of observation after administration.

The present study was not without limitations. The most notable one was the lack of an intranasal placebo as a comparator, which was not permitted by our institution's Human Research Ethics Committee because the intranasal route is a relatively new route of drug administration. Other than that, the study was limited due to its open-label nature. The patients knew the medication(s) that would be administered to them and the investigators knew the medication(s) allocated to the patients. However, the patients only knew the medication(s) allocated to them and not the medication(s) received by the patients in the other treatment arm. The results of the study may also not be generalisable, as it was a single-centre study involving a small sample size. Furthermore, the patients were recruited via convenience sampling. In addition to the aforementioned limitations, the dose of tramadol used in the study was relatively high, and it is possible that the analgesic effect from the tramadol alone was sufficient to relieve the patient's pain.

In conclusion, although intranasal fentanyl is possibly an effective analgesic alternative, we cannot be certain whether it is a safe option due to its side effects of hypotension and dizziness. The present study did not examine whether the side effects of hypotension and dizziness were clinically significant and sustained beyond the initial ten minutes of observation. The small sample size may also have introduced some response bias. Thus, larger randomised double-blind studies on the efficacy and safety of intranasal fentanyl as an alternative analgesic are warranted. Ideally, these future studies should include the use of an intranasal placebo so that a fair comparison can be made.

\section{REFERENCES}

1. Tanabe $P$, Buschmann M. A prospective study of ED pain management practices and the patient's perspective. J Emerg Nurs 1999; 25:171-7.

2. Motov SM, Khan AN. Problems and barriers of pain management in the emergency department: are we ever going to get better? J Pain Res 2008; 2:5-11.

3. Hwang U, Richardson LD, Sonuyi TO, Morrison RS. The effect of emergency department crowding on the management of pain in older adults with hip fracture. J Am Geriatr Soc 2006; 54:270-5.

4. Prommer E, Thompson L. Intranasal fentanyl for pain control: current status with a focus on patient considerations. Patient Prefer Adherence 2011; 5:157-64.

5. Wolfe TR, Braude DA. Intranasal medication delivery for children: a brief review and update. Pediatrics 2010; 126:532-7.

6. Merkus FW, Verhoef JC, Schipper NG, Marttin E. Nasal mucociliary clearance as a factor in nasal drug delivery. Adv Drug Deliv Rev 1998; 29:13-38.

7. Shelley K, Paech MJ. The clinical applications of intranasal opioids. Curr Drug Deliv 2008; 5:55-8.

8. Leonard AK, Sileno AP, Brandt GC, et al. In vitro formulation optimization of intranasal galantamine leading to enhanced bioavailability and reduced emetic response in vivo. Int J Pharm 2007; 335:138-46. 
9. Hansen MS, Dahl JB. Limited evidence for intranasal fentanyl in the emergency department and the prehospital setting--a systematic review. Dan Med J 2013; 60:A4563.

10. Borland ML, Bergesio R, Pascoe EM, Turner S, Woodger S. Intranasal fentanyl is an equivalent analgesic to oral morphine in paediatric burns patients for dressing changes: a randomised double blind crossover study. Burns 2005; 31:831-7.

11. Lee CR, McTavish D, Sorkin EM. Tramadol. A preliminary review of it pharmacodynamic and pharmacokinetic properties, and therapeutic potential in acute and chronic pain states. Drugs 1993; 46:313-40.

12. Smith S, Scarth E, Sasada M. Drugs in Anaesthesia and Intensive Care. $4^{\text {th }}$ ed. Oxford: Oxford University Press, 2011.

13. Clavijo CF, Lynn RR, Christians U, Galinkin JL. Intranasal fentanyl for breakthrough pain control. Clin Med Insights: Therapeutics 2012; 4:241-50.

14. Rickard C, O'Meara P, McGrail M, et al. A randomized controlled trial of intranasal fentanyl vs intravenous morphine for analgesia in the prehospital setting. Am J Emerg Med 2007; 25:911-7.

15. Bendall JC, Simpson PM, Middleton PM. Effectiveness of prehospital morphine, fentanyl, and methoxyflurane in pediatric patients. Prehosp Emerg Care 2011; 15:158-65.
16. Younge PA, Nicol MF, Kendall JM, Harrington AP. A prospective randomized pilot comparison of intranasal fentanyl and intramuscular morphine for analgesia in children presenting to the emergency department with clinical fractures. Emerg Med 1999; 11:90-4.

17. Borland M, Jacobs I, King B, O'Brien D. A randomized controlled trial comparing intranasal fentanyl to intravenous morphine for managing acute pain in children in the emergency department. Ann Emerg Med 2007; 49:335-40.

18. Saunders M, Adelgais K, Nelson D. Use of intranasal fentanyl for the relief of pediatric orthopedic trauma pain. Acad Emerg Med 2010; 17:1155-61.

19. Dupont WD, Plummer WD Jr. Power and sample size calculations. A review and computer program. Control Clin Trials 1990; 11:116-28.

20. Ahmad R, Mohd GA, Mohamad N, et al. A study on the effectiveness of intravenous tramadol in the management of severe traumatic pain in emergency department. J Hainan Med Coll 2010; 16:838-41.

21. Jensen MP, Chen $C$, Brugger AM. Interpretation of visual analog scale ratings and change scores: a reanalysis of two clinical trials of postoperative pain. J Pain 2003; 4:407-14.

22. Kim HY. Statistical notes for clinical researchers: assessing normal distribution (2) using skewness and kurtosis. Restor Dent Endod 2013; 38:52-4. 